\title{
Alterações otorrinolaringológicas na síndrome da apneia obstrutiva do sono
}

\author{
Maria Helena de Araújo-Melo,,${ }^{1,2,4 *}$ Lúcia Joffily, ${ }^{1}$ Lucas N. A. Lemes, $, 3,5$ Priscila S. Dias, ${ }^{3}$ Manuela S. Mosciaro ${ }^{6}$
}

\section{Resumo}

A avaliação otorrinolaringológica tem grande importância na pesquisa de alterações anatômicas em indivíduos com suspeita de síndrome da apneia obstrutiva do sono(SAOS), cuja gênese multifatorial tem uma abordagem multidisciplinar, mas é necessária sempre uma adequada visão anatômica das vias aéreas superiores (VAS). Esta avaliação visa compreender melhor as características da doença e, assim, propor o melhor tipo de tratamento individualmente. Aqui é dada ênfase ao exame antropométrico destes pacientes, à avaliação da orofaringe, da hipofaringe e da cavidade nasal, usando, de preferência, métodos endoscópicos para uma melhor observação. São valorizados critérios observados pela classificação de Mallampati (relação entre base da língua e da faringe) e de Brodsky (tamanho das tonsilas palatinas), assim como pela classificação odontológica de Angle.

Descritores: Distúrbios do sono; Apneia do sono tipo obstrutiva; Otolaringologia; Exame físico; Faringe; Cavidade nasal.

\section{Abstract \\ Otorhinolaryngologic alterations in the obstruc- tive sleep apnea syndrome}

The ENT evaluation is very important in anatomical evaluation in suspected obstructive sleep apnea syndrome (OSAS) patients, whose multifactorial syndrome genesis have multidisciplinary approach but always needs a proper anatomical view of the upper airway (UA). This review aims to better understand the anatomical characteristics of the disease and thus offer the best type of treatment individually. Here it is emphasized the anthropometric examination of these patients, evaluation of the oropharynx, hypopharynx and nasal cavity, preferably by endoscopic methods for better observation. The best criteria used are by Mallampati classification (ratio base of the tongue and pharynx) and Brodsky (size of the tonsils), as well as the dental classification Angle. It was not reviewed, in this study, any additional imaging evaluation methods.

Keywords: Sleep disorders; Obstructive sleep apnea; Otolaryngology; Physical examination; Pharynx; Nasal cavity.

\section{Resumen}

Cambios otorrinolaringológicos en el síndrome de apnea obstructiva del sueño

La evaluación ENT es muy importante en la investigación de
1. Serviço de Otorrinolaringologia. Hospital Universitário Gaffrée e Guinle. Universidade Federal do Estado do Rio de Janeiro. Rio de Janeiro, RJ, Brasil.

2. LabSono. Hospital Universitário Gaffrée e Guinle. Universidade Federal do Estado do Rio de Janeiro. Rio de Janeiro, RJ, Brasil.

3. Serviço de Otorrinolaringologia. Hospital Universitário Pedro Ernesto. Universidade do Estado do Rio de Janeiro. Rio de Janeiro, RJ, Brasil.

4. Escola de Medicina e Cirurgia. Universidade Federal do Estado do Rio de Janeiro. Rio de Janeiro, RJ, Brasil.

5. Departamento de Especialidades Cirúrgicas. Faculdade de Ciências Médicas. Universidade do Estado do Rio de Janeiro. Rio de Janeiro, RJ Brasil.

6. Serviço de Otorrinolaringologia. Hospital das Forças Aéreas do Galeão. Rio de Janeiro, RJ, Brasil

*Endereço para correspondência:

PPGNEURO, UNIRIO

Rua Mariz e Barros, 775

Rio de Janeiro, RJ, Brasil. CEP: 20270-004.

E-mail: mestneuro@gmail.com

Revista HUPE, Rio de Janeiro, 2016;15(1):44-48

doi: $10.12957 /$ rhupe.2016.22360

Recebido em 15/01/2015. Aprovado em 22/03/2016.

las alteraciones anatómicas en las personas con síndrome de apnea obstructiva del sueño (SAOS), cuya génesis multifactorial tener un enfoque multidisciplinario, pero siempre necesita una visión anatómica adecuada de la vía aérea superior (VAS). Esta revisión tiene como objetivo conocer mejor las características de la enfermedad y así ofrecer el mejor tipo de tratamiento individual. Aquí se pone de relieve el examen antropométrico de estos pacientes, la evaluación de la orofaringe, hipofaringe y la cavidad nasal, de preferencia por métodos endoscópicos para una mejor observación. Se valoran criterios utilizados por la clasificación Mallampati (relación de base de la lengua y la faringe) y Brodsky (tamaño de las amígdalas), así como el ángulo de clasificación dental. No fueron revisados, en este estudio, cualquiera de los métodos de evaluación de imagen adicionales.

Palabras clave: Trastornos del sueño; Apnea del sueño obstructiva; Otolaríngologia; Examen físico; Faringe; Cavidad nasal. 


\section{Artigo de revisão}

\section{Introdução}

O otorrinolaringologista tem grande importância no grupo multidisciplinar que avalia indivíduos com suspeita de Síndrome da Apneia Obstrutiva do Sono (SAOS). Pela pesquisa de alterações anatômicas através do exame otorrinolaringológico, são identificadas estruturas e anormalidades que possam contribuir para o estreitamento ou aumento da resistência das vias aéreas superiores (VAS) durante o sono. Dessa forma, uma avaliação otorrinolaringológica completa, por meio de exame físico sem instrumentos óticos e da endoscopia de VAS, preferencialmente usando a nasofibrolaringoscopia com fibra ótica flexível, visa identificar se as alterações anatômicas estão relacionadas com a presença de SAOS. Esta avaliação anatômica tem sido estudada por diversos autores ${ }^{1,2,3} \mathrm{e}$ é considerada indispensável para uma boa avaliação destes pacientes.

Apesar disso, sabe-se que a gênese da SAOS é multifatorial. A diferença de pressão intra e extraluminal, tônus vasomotor da musculatura faríngea e forças adesivas agindo sobre a mucosa podem colaborar com o estreitamento ou um colapso da VAS. ${ }^{4,5}$ Não só fatores anatômicos atuam nesta complexa relação mecânica, como se pode observar em doenças endócrinas (hipotireoidismo) e metabólicas (obesidade) influencim a propensão a um evento obstrutivo na SAOS. A redução do fluxo aéreo promove, através de estímulos a receptores na mucosa faríngea, breves despertares durante o sono, levando a uma fragmentação na sua continuidade que causa sonolência excessiva diurna e diversos outros sintomas clínicos e neurocognitivos associados a SAOS.

\section{Avaliação otorrinolaringológica}

Durante a avaliação clínica inicial do paciente com diagnóstico ou suspeita de SAOS, o otorrinolaringologista deve realizar, caso isto ainda não tenha sido feito, a aferição de medidas antropométricas gerais, como o IMC (índice de massa corporal) e as circunferências cervical e abdominal. Já o exame físico otorrinolaringológico será realizado com o paciente em posição sentada com a cabeça relaxada e olhando para frente. É importante aferir as cavidades nasal, oral, faríngea e a laringe. Deve-se iniciar com exame direto pela ectoscopia cervical, orofaringoscopia e rinoscopia anterior, seguido do exame endoscópico. A anestesia tópica e a vasoconstrição devem ser ajustadas aos objetivos de cada exame. No caso da endoscopia nasal, por exemplo, o uso de vasoconstrictor na cavidade pode mascarar uma hipertrofia de corneto inferior.

\section{Cavidade oral, faríngea e laríngea}

Na avaliação da cavidade oral, pede-se que o paciente simplesmente abra a boca, mantendo a língua no seu interior. São observados os elementos dentários, a sua relação entre si, o aspecto e posição das pregas na mucosa oral e o tamanho da língua e dos tecidos linfoides. Esta avaliação pode sugerir uma anatomia desproporcional da cavidade orofaríngea, obtida especificamente pela classificação de Mallampati (relação entre base da língua e da faringe) e de Brodsky (tamanho das tonsilas palatinas).

Pelo Mallampati Modificado ${ }^{6}$ (Figura1), realiza-se a orofaringoscopia com a língua dentro da boca em posição relaxada, classificada nos seguintes itens:

Grau 1 - quando os pilares amigdalianos, o palato mole e a úvula estão visíveis;

Grau 2 - quando o palato mole e a úvula estão visíveis;

Grau 3 - quando o palato mole e a base da úvula estão visíveis;

Grau 4 - quando o palato mole não está visível.

Já a classificação de Brodsky ${ }^{8}$ leva em consideração o tamanho das tonsilas palatinas e o grau de obstrução por elas exercido na orofaringe (Figura 2):

Grau 1 - as tonsilas ocupam 25\% do espaço entre os pilares amigdalianos;

Grau 2 - as tonsilas ocupam de 25\% a 50\% do espaço entre os pilares amigdalianos;

Grau 3 - as tonsilas ocupam de 50\% a 70\% do espaço
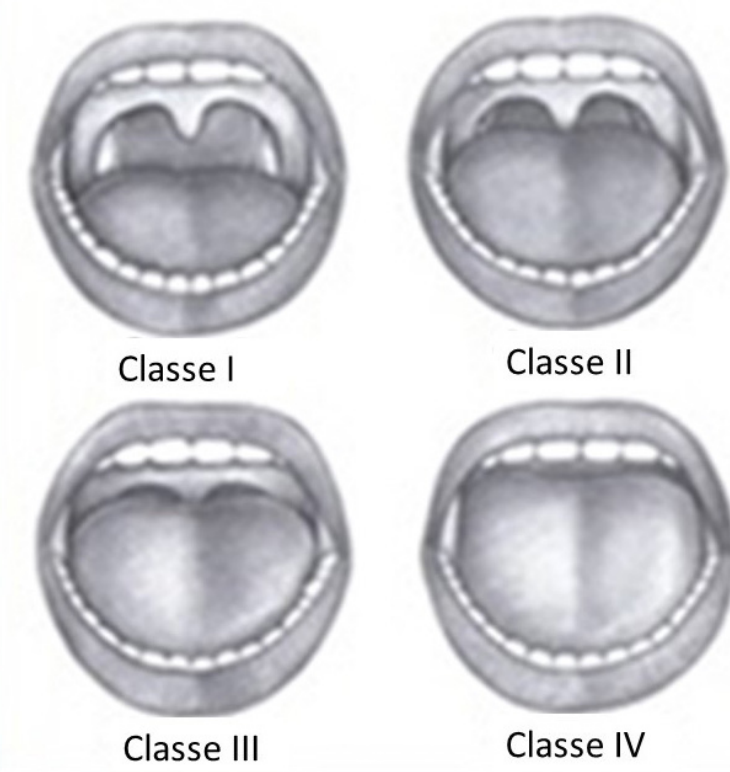

Classe II



Classe IV

Figura 1. Classificação de Mallampati modificada. 
entre os pilares amigdalianos;

Grau 4 - as tonsilas ocupam $75 \%$ ou mais do espaço entre os pilares amigdalianos.

Devem ser avaliadas também a oclusão dentária e as alterações de desenvolvimento da maxila e da mandíbula. A presença de retrognatia ou hipoplasia maxilar pode contribuir para o estreitamento da via aérea superior e predispor à obstrução dessa região durante o sono. A avaliação da oclusão dentária pode ser feita pela classificação de Angle (Figura 3). Nesta é observada a mordida do paciente e considera-se:

Classe I: adequada relação entre a arcada dentária superior e inferior;

Classe II: arcada dentária inferior retroposicionada em relação à superior - sugestivo de retroposição mandibular;

Classe III: arcada dentária inferior projetada anteriormente em relação à superior - sugestivo de prognatismo mandibular e/ou hipoplasia maxilar.

Já na orofaringoscopia, a hipertrofia das tonsilas palatinas, o palato duro ogival, a presença de palatoweb (membrana mucosa ao lado da úvula pela inserção baixa dos pilares amigdalianos posteriores), a úvula alongada e/ou espessada, o alongamento do palato mole, a centralização dos pilares amigdalianos e os pilares amigdalianos posteriores redundantes e flácidos são achados frequentes no paciente com SAOS. Esses achados são muito importantes na indicação de pro-

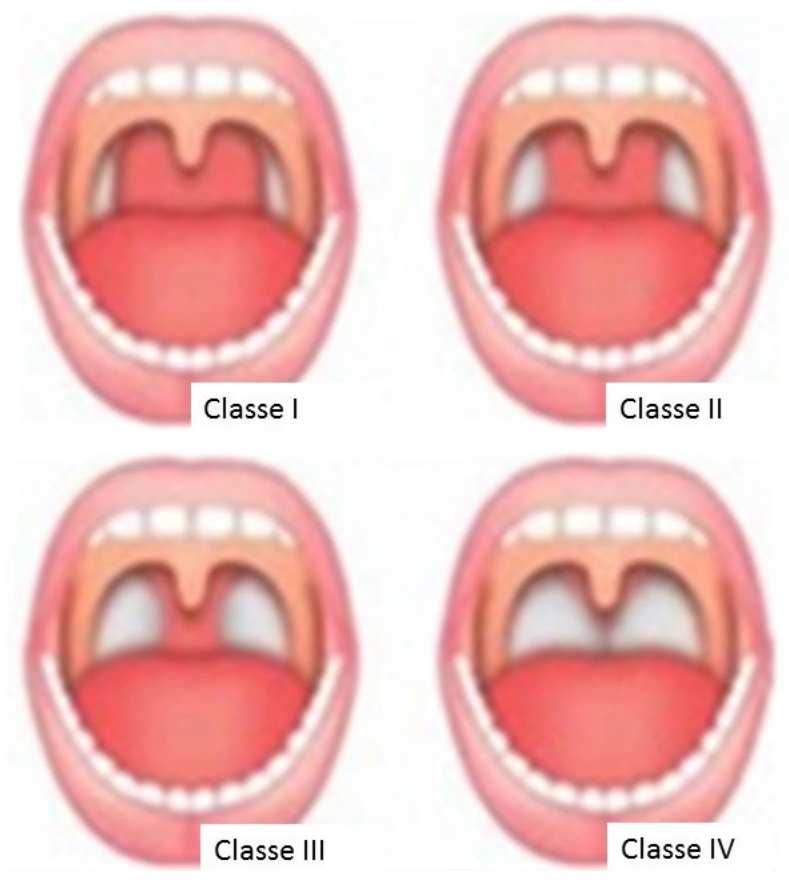

Figura 2. Classificação de Brodsky. ${ }^{8}$ cedimentos cirúrgicos para o tratamento da doença.

A avaliação endoscópica pode ser feita com endoscópios rígidos ou flexíveis visando procurar os possíveis locais de obstrução, sendo que o exame com o aparelho flexível, em geral, causa menos desconforto no paciente e permite a avaliação desde a rinofaringe até a laringe. O formato circular da faringe, a projeção da úvula, das tonsilas palatinas ou do complexo glosso-epiglótico no sentido da parede posterior da faringe são os principais parâmetros observados. A projeção está presente quando, com o endoscópio localizado na rinofaringe com sua angulação inclinada para baixo, uma porção considerável da área faríngea está ocupada por uma das estruturas acima citadas, sugerindo uma possível obstrução parcial ou total da via aérea durante o sono.

Em um estudo com 66 indivíduos, correlacionou alterações anatômicas endoscópicas com a polissonografia dos pacientes. ${ }^{9}$ A análise concluiu que o formato circular da laringe tem associação com a gravidade da doença e que a projeção da úvula no sentido da parede posterior da faringe também apresentou relação com a SAOS. Dentre os parâmetros endoscópicos, outros pontos avaliados e que não apresentaram associação foram: a projeção tonsilar e a projeção do complexo glossoepiglótico em direção à faringe, como demonstrado na tabela 1.

Sabemos que o formato fisiológico da faringe é elíptico sendo maior o seu diâmetro latero-lateral.Com

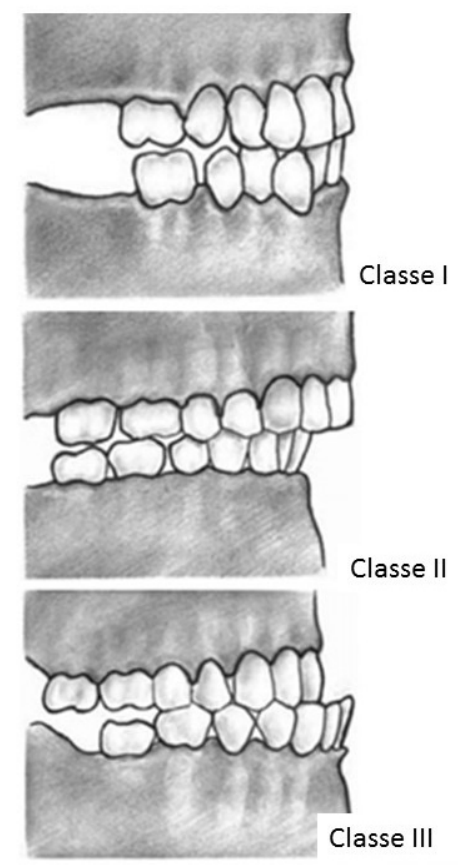

Figura 3. Classificação de Angle. 


\section{Artigo de revisão}

Tabela 1. Associação entre as alterações anatômicas Orofaringolaringológicas e SAOS. Odds Ratio ajustado (ORa) por regressão logística, Intervalos de confiança e p-valor.

\begin{tabular}{|c|c|c|c|c|c|}
\hline Variável & $\begin{array}{c}\text { Sem SAOS } \\
\text { (18 casos) } \\
(\mathrm{n} / \%)\end{array}$ & $\begin{array}{l}\text { SAOS } \\
\text { (48 casos) } \\
(\mathrm{n} / \%)\end{array}$ & ORa & p-valor & IC 95\% \\
\hline $\begin{array}{l}\text { Faringe } \\
\text { Circular } \\
\text { Elíptica }\end{array}$ & $\begin{array}{c}3(16,7) \\
15(83,3)\end{array}$ & $\begin{array}{l}13(27,1) \\
35(72,9)\end{array}$ & 1,85 & 0,379 & $0,41-11,5$ \\
\hline $\begin{array}{l}\text { Projeção Tonsilar } \\
\text { Sim } \\
\text { Não }\end{array}$ & $\begin{array}{c}2(11,1) \\
16(88,9)\end{array}$ & $\begin{array}{c}9(18,8) \\
39(81,3)\end{array}$ & 1,84 & 0,45 & $0,32-19,26$ \\
\hline $\begin{array}{l}\text { Projeção Úvula } \\
\text { Sim } \\
\text { Não }\end{array}$ & $\begin{array}{c}4(22,2) \\
14(77,8)\end{array}$ & $\begin{array}{l}28(58,3) \\
20(41,7)\end{array}$ & 4,9 & 0,008 & $1,25-22,9$ \\
\hline $\begin{array}{l}\text { Projeção CGE } \\
\text { Sim } \\
\text { Não }\end{array}$ & $\begin{array}{c}2(11,1) \\
16(88,9)\end{array}$ & $\begin{array}{l}13(27,1) \\
35(72,9)\end{array}$ & 2,9 & 0,16 & $0,55-29,7$ \\
\hline $\begin{array}{l}\text { Escore de Mallampati* } \\
\text { Alterado } \\
\text { (classes } 2,3,4) \\
\text { Normal (classe } 1 \text { ) }\end{array}$ & $\begin{array}{l}15(83,3) \\
3(16,7)\end{array}$ & $\begin{array}{l}43(89,6) \\
5(10,4)\end{array}$ & 1,72 & 0,48 & $0,23-10,05$ \\
\hline $\begin{array}{c}\text { Classificação de Brodsky* } \\
\text { Classes 2,3,4 } \\
\text { Classes 0,1 }\end{array}$ & $\begin{array}{c}6(33,3) \\
12(66,7)\end{array}$ & $\begin{array}{l}16(33,3) \\
32(66,7)\end{array}$ & 1,00 & 1,0 & $0,28-3,87$ \\
\hline
\end{tabular}

Legenda: $\mathrm{SAOS}=$ Síndrome da Apneia do Sono, $\mathrm{n}=$ número, \%= por cento, $\mathrm{p}=\mathrm{p}$-valor, ORa= OddsRatio Ajustado, IC= intervalo de confiança.

Tabela 2. Distribuição das alterações nasais segundo os grupos do IAH (1= IAH até 5; 2= IAH 5-15; 3= IAH 15-30; 4= IAH > 30).

\begin{tabular}{|c|c|c|c|c|c|c|c|}
\hline \multirow{2}{*}{ Variável } & \multirow{2}{*}{ Qualificação } & \multicolumn{4}{|c|}{ Grupos } & \multirow{2}{*}{$\begin{array}{c}\text { Total } \\
(n=63 / \%)\end{array}$} & \multirow{2}{*}{$P$ valor } \\
\hline & & $1(n=17)$ & $2(n=19)$ & $3(n=14)$ & $4(n=13)$ & & \\
\hline \multirow{2}{*}{ Obstrução Nasal } & Presente & 11 & 14 & 8 & 9 & $42(66,7 \%)$ & \multirow{2}{*}{0,7867} \\
\hline & Ausente & 6 & 5 & 6 & 4 & $21(33,3 \%)$ & \\
\hline \multirow{2}{*}{$\begin{array}{l}\text { Conchas Inferiores } \\
\text { Hipertróficas }\end{array}$} & Presente & 14 & 16 & 13 & 12 & $55(87,3 \%)$ & \multirow{2}{*}{0,214} \\
\hline & Ausente & 3 & 3 & 1 & 1 & $8(12,7 \%)$ & \\
\hline \multirow{2}{*}{ Desvio obstrutivo } & Presente & 11 & 8 & 10 & 9 & 37 (58.7\%) & \multirow{2}{*}{0,6962} \\
\hline & Ausente & 6 & 11 & 4 & 5 & $26(41,3 \%)$ & \\
\hline \multirow{2}{*}{ Desvio não obstrutivo } & Presente & 4 & 11 & 3 & 4 & $22(34,9 \%)$ & \multirow{2}{*}{0,0857} \\
\hline & Ausente & 13 & 8 & 11 & 9 & $41(65,1 \%)$ & \\
\hline
\end{tabular}

Legenda: $\mathrm{AlH}=$ índice de apneia, $\mathrm{n}=$ número, \%= por cento, $\mathrm{p}=$ pvalor.

a evolução da doença, a faringe assume incialmente um formato circular e, em fases mais avançadas, volta a ter um formato elíptico, porém sendo maior o diâmetro antero-posterior devido à hipertrofia da musculatura lateral faríngea. Esse raciocínio vai de acordo com a relação encontrada entre o formato da faringe e a gravidade da SAOS.

\section{Cavidade nasal}

As alterações estruturais da cavidade nasal são avaliadas pelo exame fisico com espéculo nasal (rinoscopia anterior) e por endoscópios rígidos ou flexíveis. Os parâmetros a serem observados são a presença de desvio de septo, hipertrofia de conchas nasais e hipertrofia das tonsilas faríngeas no nasofaringe. 
A presença de alterações estruturais na cavidade nasal tem grande prevalência em pacientes com SAOS, pois estas alterações podem levar a obstrução do fluxo aéreo nasal e, consequentemente, aumentar a sua resistência. ${ }^{3} \mathrm{Na}$ literatura, foi descrito que, durante o sono, há um aumento na resistência nasal causado pela posição supina e pela diminuição do tônus muscular. ${ }^{10}$

Foi observada semelhança na prevalência de hipertrofia de conchas nasais inferiores e desvio septal obstrutivo entre os grupos de pacientes apneicos e o grupo controle $(\mathrm{IAH}<5 / \mathrm{h}){ }^{9}$ não havendo assim diferença significativa em relação à distribuição destas alterações, nem do sintoma obstrução nasal, em relação à gravidade da SAOS medida pelo IAH (Tabela 2).

As outras variáveis estudadas foram tonsilas faríngeas presentes no cavum, desvio septal de acordo com a classificação pelas áreas de Cottle (uni ou bilateral) e hipertrofia das conchas nasais inferiores (uni ou bilaterais), todas sem significância estatística.

Outras condições de obstrução nasal reversível, como Rinite Alérgica, já foram descritas em outros trabalhos, ${ }^{11,12}$ sendo consideradas um fator contribuinte no desenvolvimento da SAOS.

\section{Sonoendoscopia}

A sonoendoscopia é um dos métodos mais recentes de avaliação do paciente com SAOS e é realizada com este em decúbito dorsal sob indução anestésica, com sedativos como propofol, com o objetivo de induzir um sono artificial para a realização da endoscopia. $\mathrm{O}$ objetivo deste exame seria observar o exato ponto de colapso na via aérea do paciente durante o sono. Esse exame, contudo, necessita de cuidados específicos do ponto de vista de segurança para o paciente. Além disso, o sono induzido por anestésicos afeta os mecanismos de manutenção da patência da via aérea, o que pode levar a uma interpretação errada do que ocorreria em uma noite de sono natural do paciente..$^{13}$ Devido a esses motivos, não há um consenso em relação à avaliação do paciente apneico com este método, sendo necessários mais estudos que comprovem a sua validade.

\section{Considerações finais}

O otorrinolaringologista tem uma participação de extrema importância no manejo da SAOS, sendo recomendado, inclusive, que o exame físico otorrino- laringológico seja realizado antes da polissonografia. Entretanto, em muitas situações, é necessária também a intervenção de outros profissionais, inclusive de dentistas e fisioterapeutas.

A SAOS é uma doença de cunho multifatorial e, como tal, há necessidade de abordagem multidisciplinar. É fundamental que cada profissional faça uma avaliação completa dentro da sua área de atuação e, dessa forma, o paciente se beneficie ao máximo de cada especialidade.

\section{Referências}

1. Neto LM, Fava AS, Lopes HC, et al. Estudo epidemiológico das alterações estruturais da cavidade nasal associadas à síndrome da apneia e hipopneia obstrutiva do sono (SAHOS). Rev Bras Otorrinolaringol. 2005;71(4):464-6.

2. Series F, Pierre SS, Carrier G. Effects of surgical correction of nasal obstruction in the treatment of obstructive sleep apnea. Am Rev Resp Dis. 1992;145:1261-5.

3. Pang KP, Terris DJ. Severity of obstructive sleep apnea: Correlation with clinical examination and patient perception. Otolaryngol Head Neck Surg. 2006;135(4):555-60.

4. Haddad FL, Vidigal TD, Mello-Fujita L, et al. The influence of nasal abnormalities in adherence to continuous positive airway pressure device therapy in obstructive sleep apneia patients. Sleep Breath. 2013;17:1201-7.

5. Soares MCM, Bittencourt LRA, Zonato AL, et al. Aplicação do modelo morfométrico de Kushida em pacientes com distúrbios respiratórios do sono. Rev Bras Otorrinolaringol. 2006;72(4):541-8.

6. Kim J, In K, Kim J, et al. Prevalence of sleep-disordered in middle-aged Korean men and women. Am J resp Crit Care Med. 2004;170(10):1108-13.

7. Bittencourt LRA, Haddad FM, Fabbro CD, et al. Abordagem Geral do Paciente com Síndrome da Apneia Obstrutiva do Sono. Rev Bras Hipertens. 2009;15(3):158-63.

8. Young T, Hutton R, Finn L, et al. The gender bias in sleep apnea diagnosis: are women missed because they have different symptoms? Arch Intern Med 1996;156(2):2445-51.

9. Dias PS, Araujo-Melo MH, Neves DD, et al. Correlação entre os achados orofaringolaringoscópicos e a gravidade da síndrome da apneia obstrutiva do sono Rev Col Bras Cir. 2015;42(5):28929.

10. McNicholas W, Coffey M, Boyle T. Effects of nasal airflow on breathing during sleep in normal humans. Am Rev Respir Dis. 1993;147:620-3.

11. Young T, Finn L, Kim H. Nasal obstruction as a risk factor for sleep disordered breathing. J Allergy Clin Immunol. 1997;99:S757-S762.

12. Sogebi OA, Ogunwala A. Risk factors of obstructive sleep apnea among nigerian outpatients. Braz J Otorhinolaryngol. 2012;78(6):27-33.

13. Kryger MH, Roth T, Dement WC, eds. Principles and practice of Sleep Medicine. Philadelphia: WB Sauders Company; 2010. $1216 \mathrm{p}$. 